\title{
Coexistence of 2 rare autosomal recessively inherited disorders manifesting with immune deficiency; IL-12 receptor $\beta 1$ and biotinidase deficiencies
}

\author{
Dilek Doğruel ${ }^{1}$, Fatma Derya Bulut ${ }^{2}$, Mustafa Yılmaz ${ }^{1}$, Neslihan Önenli-Mungan², \\ Derya Ufuk Altıntaş ${ }^{1}$ \\ ${ }^{1}$ Division of Allergy and Immunology and ${ }^{2}$ Metabolism and Nutrition, Department of Pediatrics, Çukurova University \\ Faculty of Medicine, Adana, Turkey. E-mail: dilekkaragoz1977@hotmail.com \\ Received: 30th May 2017, Revised: 1st August 2017, Accepted: 8th October 2017
}

\begin{abstract}
SUMMARY: Doğruel D, Bulut FD, Yılmaz M, Önenli-Mungan N, Altıntaş DU. Coexistence of 2 rare autosomal recessively inherited disorders manifesting with immune deficiency; IL-12 receptor $\beta 1$ and biotinidase deficiencies. Turk J Pediatr 2018; 60: 584-587.

In this report, we described an infant with both partial biotinidase and IL$12 \mathrm{R} \beta 1$ deficiencies as these two entities are rare and unrelated inherited disorders. One-month-old girl was diagnosed as partial biotinidase deficiency with newborn screening programme. Mutation analysis revealed a compound heterozygous mutation BTD: c.1330G >C (p.Val444Leu) / c.196 197dupCATC (p.Leu69HisfsTer24). At the age of 6 months, a nodule on her left axilla with purulent discharge was noticed which was related to BCG vaccination. A mutational analysis revealed a homozygous c. $783+1 \mathrm{G}>\mathrm{A}$ mutation on $I L$ $12 R \beta 1$ gene. Interferon-gamma and anti-tuberculosis treatment were initiated together and the nodule with purulent discharge regressed dramatically. Here, we want to emphasize consideration of coexistence of two rare autosomal recessively inherited diseases in a patient due to the high rate of consanguinity in our country.
\end{abstract}

Key words: BCG, lymphadenitis, biotinidase deficiency, IL-12R $\beta 1$ deficiency, MSMD.

Mendelian susceptibility to mycobacterial infections (MSMD) is an autosomal recessively inherited rare disease. Patients are susceptible to infections due to BCG (Bacilli Calmette-Guerin) vaccination, non-tuberculosis mycobacteria, and also Salmonella strains. IL-12 receptor beta 1 (IL-12R $\beta 1$ ) deficiency is the most common form of MSMD and disease spectrum varies between local infections to systemic BCG infection. ${ }^{1}$

Biotin is a vitamin that works as a cofactor of carboxylases in the organism. Biotinidase deficiency (BD) is an autosomal recessively inherited metabolic disease caused by disturbed recycling of biotin, leading to metabolic acidosis, cutaneous and neurological manifestations together with specific laboratory signs. ${ }^{2}$ Additionally, impaired $\mathrm{T}$ cell function causes immune deficiency and lead to recurrent infections. ${ }^{3}$ As a result of high consanguinity rate, $\mathrm{BD}$ is a frequently seen as inborn error of metabolism in Turkey. ${ }^{4}$

Here, we present an infant with BCG lymphadenitis (BCG-itis) due to IL-12R $\beta 1$ deficiency caused by a homozygous mutation in the IL12Rß1 gene and partial BD due to compound heterozygous mutation in the BTD gene.

\section{Case Report}

A 1-month-old girl was referred to our hospital for the confirmation of BD which was detected by the national newborn screening program. She was born 3300 grams, at 39 weeks of gestation without a problem. Her parents were first degree cousins and she had a healthy brother. Otherwise the family history was unremarkable for an inherited disease, immune deficiencies or child death with an unknown

This study was presented at the 3th Clinical Immunology Congress, 12-15 April 2017. 
cause. Umbilical cord separation occurred on fifth day of life. Her growth parameters, neuromotor development, and physical examination findings were fully normal for her age. She had no clinical symptoms or signs of BD. Serum biotinidase activity was measured as $2.39 \mathrm{U} / \mathrm{L}(\mathrm{N}: 3.8-13.3)$. Biotin replacement was started at a dose of $5 \mathrm{mg} /$ day for partial BD. Serum amino acids, acylcarnitine profile and urinary organic acid analysis were normal. Molecular analysis detected a compound heterozygous mutation in the BTD gene: NM_001281724.2 c.1330G >C (p.Val444Leu) / c.196_197dupCATC (p.Leu69HisfsTer24) (Fig. 1). With biotin replacement therapy she was healthy until the age of six months, when the family was first aware of a lymphadenopathy with a diameter of $2 \times 2 \mathrm{~cm}$ in her left axillary region which was persisting for two weeks. Detailed examination of the patient showed purulent discharge from the BCG scar on her left shoulder with multiple lymph nodes and a lesion consistent with an abscess up to 2 $\mathrm{cm}$ in diameter in the left axillary region. She had been vaccinated with BCG at the age of 2 months. Laboratory examination revealed a mild hypochromic microcytic anemia with normal results of acute phase reactants (erythrocyte sedimentation rate: $12 \mathrm{~mm} / \mathrm{h}$; C-reactive protein: $0.33 \mathrm{mg} / \mathrm{L})$. When the tuberculin test was positive $(12 \times 13 \mathrm{~mm})$, anti-mycobacterial treatment with isoniazid, rifampicin, and etambutol was initiated. The patient was also evaluated for a possible IL12R $\beta 1$ deficiency. Mutation analysis put forward a homozygous NM_005535.2 c.783+1G>A mutation on IL12R $\beta \overline{1}$ gene. Interferon-gamma (IFNy) therapy was added to the treatment. After this combined therapy the patient's lymphadenopathies, abscess, and the purulent drainage regressed dramatically. At the last visit, the patient was 1-year-old and had no health problems.

We obtained the informed consent that was obtained from the parents.

\section{Discussion}

In this report, we present an infant with co-existing, two rare hereditary disorders: biotinidase and IL-12R $\beta$ I deficiencies. The prevalence of $\mathrm{BD}$ is about 1 in 60000 live births and the prevalence of IL-12R $\beta 1$ deficiency is about 1 in 100000-1000000 births. Both BD and IL-12R $\beta 1$ deficiency are rare diseases and the probability of their co-existence is very low. Indeed, high consanguinity rates of our country may increase this possibility.

Biotinidase deficiency was first defined in biotin responsive multiple carboxylase deficiency by Gompertz et al. in 1971..$^{5}$ Consequently, BTD gene has been discovered and accepted as the responsible factor for $\mathrm{BD}$. Up to date, more than 100 pathogenic mutations on

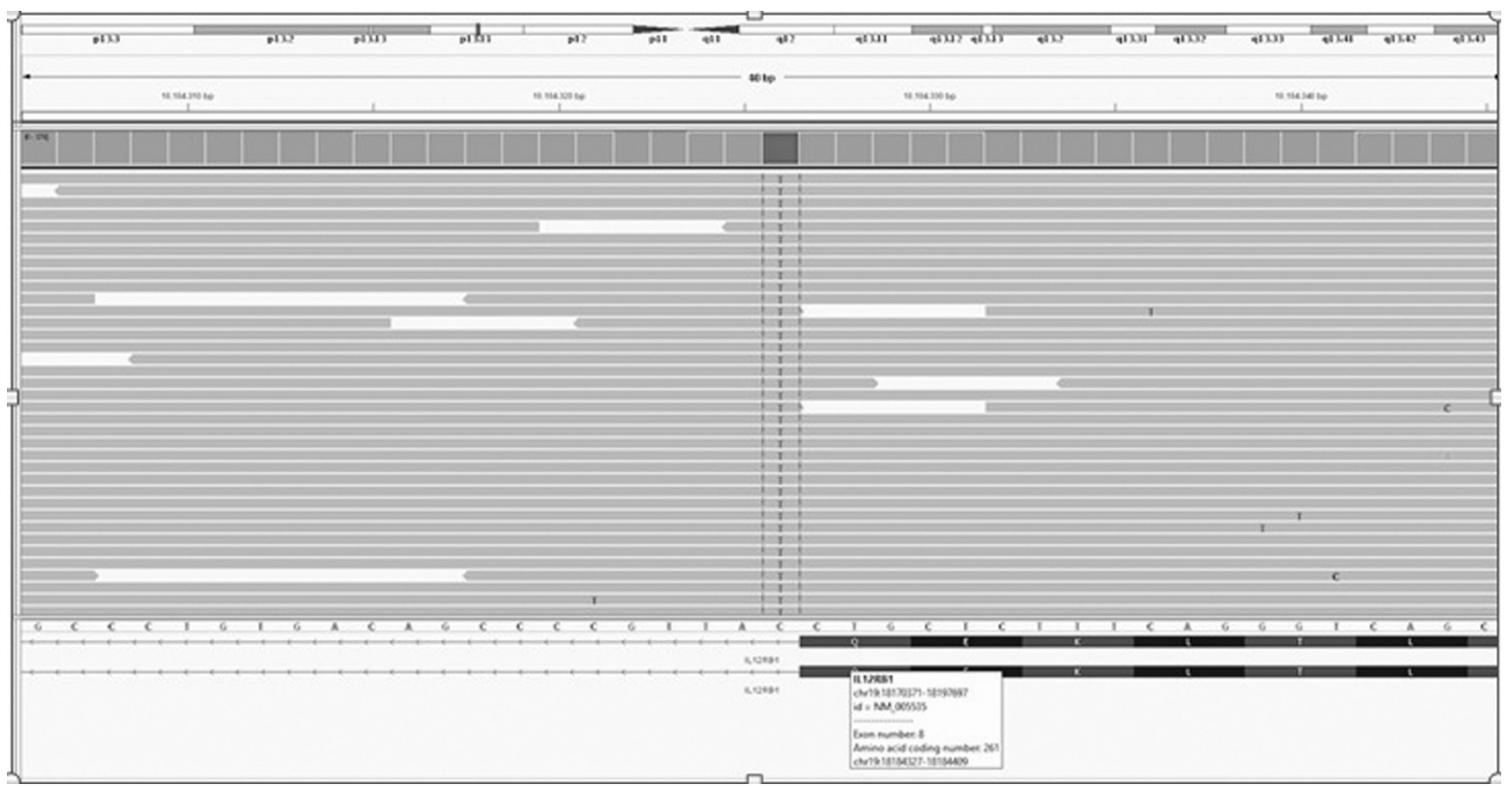

Fig. 1. A mutation in the $I L-12 R \beta 1$ gene. 
BTD gene have been described. ${ }^{6}$ The disease is characterized by convulsions, hypotonia, psychomotor retardation, progressive deafness and blindness. In addition to these clinical findings, skin lesions and infections due to immunological dysfunction are frequently seen. Signs and symptoms may begin in infancy. As late diagnosis and treatment of $\mathrm{BD}$ can cause irreversible neurological, ophthalmological damages, sensorineural hearing loss, blindness and autistic behaviors, neonatal screening programs are crucial. ${ }^{4}$ Newborn screening program for BD was initiated in 2008 in Turkey. Our patient with partial BD was detected in the first month of age before any symptoms occurred and followed as a healthy infant with $5 \mathrm{mg} / \mathrm{d}$ biotin supplementation to the age of six months when a BCG lymphadenitis was noticed. Biotinidase deficiency may cause cellular immune deficiency. ${ }^{3}$ However, the most important host defense against intracellular pathogens like Mycobacteria and Salmonella is cellular immunity and the basic mechanism is macrophage activation via type 1 cytokines. Amongst them, the most important cytokine is IFNy. T helper lymphocytes and natural killer cells produce IFNy as a consequence of IL12 binding to IL-12R $\beta 1$ and IL-12R $\beta 2$ on the cells' surface. ${ }^{1}$ So, we investigated the patient for IFNy deficiency, and found a homozygous mutation on IL12R $\beta 1$ gene. Generally, the response to antimycobacterial therapy is quite satisfactory in IL-12R $\beta 1$ deficiency. But, it is recommended to add IFNy to antimycobacterial therapy in order to shorten the treatment period and decrease the risk of tendency to recurrent mycobacterial infections with high mortality rates in symptomatic patients. Although our patient's response to antituberculosis therapy was sufficient, the additional problem of $\mathrm{BD}$, which also can lead to cellular immune dysfunction, highlighted us about adding IFNyto the treatment. To date a direct genetic link between immune disorders including IL-12R $\beta 1$ deficiency and BD has not been reported in the literature. The existing familial relationship between the parents was of note. IL12Rß1 and BTD gene was examined in the family members, and same heterozygous mutation was found in the parents. They were unaware of their condition as carriers and transmitted both diseases to their children.

As there is not any screening program for IL-
$12 \mathrm{R} \beta 1$ deficiency in the world, the diagnosis of IL-12R $\beta 1$ deficiency was frequently thought after a BCG lymphadenitis. ${ }^{7}$ Identifying the underlying problem of patients with BCG lymphadenitis is important. Because prolonged and aggressive antituberculosis treatment is essential for the patients with IL-12R $\beta 1$ deficiency. 8,9 Our patient was also diagnosed as IL-12R $\beta 1$ deficiency after a BCG lymphadenitis, and antituberculosis treatment with three drugs was initiated.

Although parents are relatives, BTD gene has compound heterozygote. This is because of high frequency of heterozygote carriers in the population. Carrier frequency in the general population is approximately one in $120 .{ }^{10}$

In conclusion, coexistence of two different hereditary disorders should be in mind, in patients when the clinical signs cannot be explained satisfactorily with one disease, especially in countries like Turkey, where parental consanguinity rate is high. One consequence of familial relationship between the parents is an increase of recessive disease. We wanted to draw attention to the coexistence of these two rare hereditary diseases that have not been previously reported in the literature. Additionally, lymphadenitis following BCG vaccination requires investigation for immune deficiencies including IL-12R $\beta 1$ deficiency. This is extremely important in countries where tuberculosis prevalence is high and BCG vaccination is routine.

\section{Acknowledgements}

We would like to thank Serdar Ceylaner for genetic analysis.

\section{REFERENCES}

1. Asilsoy S, Bilgili G, Turul T, et al. Interleukin-12/-23 receptor beta 1 deficiency in an infant with draining BCG lymphadenitis. Pediatr Int 2009; 51: 310-312.

2. Wolf B. Biotinidase deficiency: New directions and practical concerns. Curr Treat Options Neurol 2003; 5: 321-328.

3. Rezvani I, Rosenblatt DS. Valine, leucine, isoleucine, and related organic acidemias. In: Behrman RE, Kliegman RM and Jenson HB (eds). Nelson Textbook of Pediatrics (17th ed) Philadelphia: WB Saunders, 2004: 409-418.

4. Kiykim E, Kiykim A, Cansever MS, Zeybek CA. Biotinidase deficiency mimicking primary immune deficiencies. BMJ Case Rep 2015; 2015. pii: bcr2014209275. 
5. Gompertz D, Draffan GH, Watts JL, Hull D. Biotinresponsive beta-methylcrotonylglycinuria. Lancet 1971; 2: $22-24$

6. Pomponio RJ, Hymes J, Reynolds TR, et al. Mutations in the human biotinidase gene that cause profound biotinidase deficiency in symptomatic children: Molecular, biochemical, and clinical analysis. Pediatr Res 1997; 42: 840-848.

7. de Beaucoudrey L, Samarina A, Bustamante J, et al. Revisiting human IL-12R $\beta 1$ deficiency: A survey of 141 patients from 30 countries. Medicine (Baltimore) 2010; 89: 381-402.
8. Senanayake MP, Kumararatne DS, Doffinger R, BarcenasMorales G. Disseminated BCG in an infant with interleukin-12 receptor B1 (IL12RB1) deficiency. Paediatr Int Child Health 2015; 35: 69-71.

9. Tan Ç, Çağdaş-Ayvaz D, Metin A, Keskin Ö, Tezcan İ, Sanal Ö. Clinical and genetic features of IL12R $\beta 1$ deficiency: Single center experience of 18 patients. Turk J Pediatr 2016; 58: 356-361

10. https://www.ncbi.nlm.nih.gov/books/NBK1322/. 